\title{
FICOFLORA DEL SUSTRATO ROCOSO DENTRO DE LAS COSTAS DEL GOLFO DE MEXICO, MEXICO
}

\author{
Ma. ELENA SANCHEZ RODRIGUEZ
}

Escuela Nacional de Ciencias Biologicas. Instituto Politecnico Nacional, México, D.F., México

El Estado de Veracruz queda comprendido dentro de la $\mathrm{Re}$ pública Mexicana entre los $16^{\circ} 50^{\prime}$ y $22^{\circ} 10^{\prime}$ latitud $\mathrm{N}$ y los $93^{\circ} 40^{\prime}$ y $98^{\circ} 20^{\prime}$ longitud $W$. Biogeograficamente corresponde a la región Neotropical y las condiciones climáticas que prevalecen en esta región hacen que este Estado sea uno de los más ricos del País.

Durante el año de 1970, el Gobierno Federal a través de la Comisión Federal de la Electricidad, decretó el establecimiento de una planta Núcleo-Eléctrica productora dé energía, que surtirá de este elemento principalmente al Estado de Veracuz y Estados adyacentes al mismo, esta planta de acuerdo con los proyectos elaborados deberá empezar a funcionar a más tardar en el año de 1983; el área selecionada para este propósito fué Punta Limón situada ar roximadamente entre los $19^{\circ} 42^{\prime}$ latitud $\mathrm{N}$ y $96^{\circ} 24^{\prime}$ ' longitud W.

Considerando lo anterior, se dicidió hacer un estudio previo sobre la vegetación ficológica marina de las áreas rocosas del Golfo de México, visitando cinco localidades correspondientes a los estribaciones de la Cordillera Neovolcánica y tres localidades de las estribaciones de la Sierra de los Tuxtlas, los resultados obtenidos serán comparados con estudios posteriores que se realizaran en los mismos lugares después de que la planta núcleo eléctrica haya comenzado a trabajar, con el objeto de determinar si la población vegetal marina ha sufrido cambios o efectos nocivos debido al funcionamiento de la mencionada planta.

El litoral del Golgo de México, en la República Mexicana, está constituido en general por playas bajas y arenosas, excepcionaimente se presentan elevaciones rocosas originadas por: el extremo oriental de la Cordillera Neovolcánica y La Sierra de la Región de los Tuxtlas en el Estado de Veracruz; estas prominencias son de importancia cuando llegan a la orilla para constituir las costas rocosas, las cuales decienden ya sea abruptamence formando acantilados, o bien en forma lenta, lo que permite que los riscos penetren en el piélago para constituir sustratos propios para el desarrollo de algunos biotas marinos, entre ellos las algas.

Las estribaciones de la Cordillera Neovolcánica, comienzan en una pequeña área, rocosa conocida como Punta El Morro, la cual se localiza aproximadamente a $50 \mathrm{~km}$ al SE de la barra de Nautla para terminar a $100 \mathrm{~km}$ de la misma en la llamada Punta Zempoala, sin embargo, los $50 \mathrm{~km}$ de litoral rocoso no son continuos pues están interrumpidos por playas arenosas y pequeñas lagunas costeras, quedando limitada por lo tanto a pequeñas salientes que constituyen (además de las antes mencionadas) las puntas conocidas localmente como: Delgada, Limón, Villa Rica y de la Mancha.

Las estribaciones de la Sierra de los Tuxtlas practicamente comienzan en Punta Arenas mas o menos a $60 \mathrm{~km}$ at SE de la Laguna de Alvarado, para terminar en la Punta Morro situada al SE de la Laguna de Sontecomapan aproximadamente a $90 \mathrm{~km}$ de la primera laguna mencionada; el afloramiento se encuentra menos interrumpido que el de la Cordillera Neovolcánica y en él se puede distinguir además de las Puntas Arenas y Morro, las puntas Organo, Morrillo y Sontecomapan.

De la Cordillera Neovolcánica se estudiaron las siguientes localidades: Playa Morro (Punta Morro); Playa Boca Andrea (Punta Delgada); Laguna Verde (Punta Limón); Villa Rica (Punta Villa Rica) y rlaya Paraiso (Punta Mancha); cada localidad se visitó cinco veces en diferentes épocas del año; pudiendose establecer la frecuencia, abundancia y dominancia, así como la época de reproduccion de las afferentes especies que ahi se cuesarrollan.

De la parte correspondiente a la región de los Tuxtlas se han estudiado basta la fecha tres localidades: Punta Organo. Playa Monte Pfo (Punta Morrillo) y Playa Balzapote (Punta Sontecomapan); la primera localidad solo se ha visitado en una ocasión y la información que se tiene hasta el momento es poca, Playa Balza pote se visitó en una ocasión y parece ser que la vegetación es bastante pobre, debido a las razones antes expuestas estas dos localidades están excluidas en el presente trabajo. Playa Monte Pío se visitó en tres épócas diferentes y los resultados obtenidos perrhiten conpararios con los de la Cordillera del eje Neovolcánico.

Tanto en el eje Neovolcánico como en la región de los Tux. tlas, las rocas corresponden a basaltos, andesitas y tobas; en la parte correspondiente a Villa Rica y sobre todo en Playa Paraíso, abundan las areniscas cubiertas por las secreciones emenadas del poliqueto Phragmatopoma y a menudo mezclados con fragmentos de conchas varena.

Las drversas especies que crecen en estos lugares se encuentran distribuidas en los pisos, facies y modos, constrtuyendo con- dirinnes diferentes de acuerdo con las combinaciones de estos tres habitats. El piso que soporta la vegetación más variada es el litoral, el infralitoral en algunos lugares como Villa Rica y Monte Pío es practicamente inaccesible; el supralitoral solo se encuentra habitado en lugares muy sombreados y húmedos de El Morro, Boca Andrea y Laguna Verde; en las demás localidades no está representado debido posiblemente, a la exposición prolongada de luz y temperatura a los que, se encuentran sometidas las rocas. Las cubetas y pozos son frecuentes y debido a las condiciones climáticas que ahí prevalecen soportan poblaciones de algas que no se desarrollan en los lugares expuestos.

El total de especies encontrados fue de 130; el Cuadro L, corresponde a la lista florística en donde se indica además, la' o las localidades para cada especie; en el Cuadro II, se señala el número de especies pertenecientes a cada División y se considera el número de especies comunes a todas las localidades, las comunes al eje Neovolcánico y las exclusivas de cada lugar estudiado. De acuerdo con los cuadros, de las 130 especies sólo 33 son comunes a todas las localidades; 14 son comunes al eje Neovolcánico. por otra parte la vegetación es pobre y heterogenea a pesar de que entre cada una de las localidades del eje hay una distancia aproximada de $10 \mathrm{~km}$.

Las especies exclusivas de interés en El Morro son: Scinaia complanata, Rosenvigea intricata, Codium taylorii y Lythophyllum $\mathrm{sp}$; las tres primeras crecen en el piso infralitoral, facies rocosa-arenosa, modo protegido y la última se encuentra en el nivel más alto del piso litoral, facies rocosa, modo protegido en donde forma un cinturón bastante conspicuo de color rosado. Es seguro que las sugerencias que se forman en estos lúgares permitan el desarrollo de las plantas mencionadas, este mismo fenómeno podría explicar la presencia de otras especies exclusivas como sucede con Griffithsio globulifera que en Playa Paraíso convive con Wrangelia argus y Spatoglossum schoederii especies comunes al eje Neovolcánico; no hay que olvidar que las surgencias además de llevar nutrientes a la superficie suben aguas frías de los fondos y que la temperatura del agua debe depender del tipo de corrientes o capas inferiores que tal vez no sean homogeneas a lo largo de la costa.

En Villa Rica, hay en la parte más superior del piso litoral, cubetas que durante el mediodía mantienen temperaturas altas y en donde se fiian especies exchusivas de diatomeas como: Achnantes brevipes, Melosira urgensi, Melosita roseana y Nitzchua sp. Las especies de Laguna Verde se deben a la presencia de una laguna salubre muy cercana a los sitios de muestreo; en cuanto a Monte Pío la presença de las especies de Liagora, parecen estar en relación con la temperatura que en este lugar parece más estable ya que practicamente las surzencias no se manifiestan. estas especies corresponden a las formas effimeras. En ocasiones el habitat que ocupan algunas especies presenta las mismas características en dos localidades diferentes así Ulva fasciata y Enteromorpha flexuosa presentes en Monte Pío conviven en lugares semejantes a los de Ulva lactuca y Enteromorpha lingulata comunes al eje Neovolcánico. Las especies de Padina tienden' a formar asociaciones en las cubetas que se encontran al nivel medio o inferior del piso litoral, las asociaciones pueden ser de diversas especies pero los habitats son muy parecidos.

Sphacelaria tribuloides en Monte Pío, forma asociaciones dentro de las cubetas, sin embargo en el eje Neovolcánico vive como epífita de otras algas. Las asociaciones de Cymopolia barbata, comunes a todas las localidades se desarrolla gracias a la presencia de pequeñas lagunas formadas por las barreras rocosas que permiten el establecimiento del piso infralitoral, facies arenosa, modo tranquilo.

Ectocarpus oreviarticulatus y Chnoospora minima son especies que se presentan en toda el área.rocosa de Veracruz y manifiestan una clara competencia por el habitat, ambos se desarrollan en la parte superior del piso infralitoral, facies rocosa modo expuesto; $E$. breviarticulatus que tiende a disminuir su población en determinadas épocas del año forma un cinturón por arriba del de $C$. minima, cuando la población de la primera disminuye, la segunda tiende a ocupar el lugar libre, al recuperarse la población de $E$. breviarticulatus se desarrollará sobre la población de $C$. minima constituyendo la asociación Ectocarpus breviarticula. tus-Chnoospora minima, este comportamiento de $E$, breviarticulatus por el habitaculo corresponde a necesidades de mayor 
exposición a la tuz y menor a la sumerción, mientras que en $C$. minima el límite de resistencia a estos factores es más amplio.

Sargassum vulgare constituye, la asociación dominante del nivel más alto del piso infralitoral, facies rocosa, modo expuesto, frecuentemente la población manifiesta un número bastante considerable de epífitas, en los meses más cálidos del año Corallina subulata cubre casi en su totalidad las frondas de $S$. vulgare constituyendo la asaciación Sargassum vulgare-Coralina suhulata que domina durante la época mencionada; $S$. vulgare crece además en el piso infralitoral facies arenosa, modo tranquilo, en este habitat se le localiza fijo a guijarros o fragmentos de conchas, la población se reduce a un número bajo de individuos pero con frondos más extendidos y profusos, casi libres de epífitas y con pocas ramas reproductoras, por su parte $C$. subulata forma asociaciones con otras especies de algas rojas como Brythamniom triquetrum, B. seafor. thii y Digenia simplex.

En el Morro, Boca Andrea y Laguna Verde, se hallan lugares poco expuestos a la luz y sobre todo protegidos a la desecación en el piso supralitoral, donde se presenta la asociación Bostrychia radicans que forma un cinturón angosto de color rojo muy oscuro; la orientación SE que presentan aquí las rocas permite condiciones de sombra, las cuales no se manifiestan en las demás localidades, donde las neñas reciben la luz solar constante. lo aue hace aumente la temperatura de los riscos, provocando la evaporación la cual, se convierte en factor limitante para el desarrollo de algas que tienden a habitar el piso supralitoral.

En los pisos, supralitoral, litoral e infralitoral se desarrollan asociaciones de algas diversas de las cuales, algunas parecen ser comunes a toda el area rocosa; se consideran que estas asociaciones frecuentes en el área, pueden servir como índice de referencia a estudios posteriores relacionados a cambios de vegetación; además de las asociaciones mencionadas con anterioridad se señalaron los de: Hypnea musciformes-Laurencia papillosa, Ralfsia éxpansa, Bachelotia antillarum-Enteromorpha spp. y Cladophora fascicularis-Centroceras clavulatum entre las más comunes. Las cubetas litorales además de las asociaciones de Padina spp., soportan poblaciones de Colpomenia sinuosa, Laurencia obtusa, Caulerpa sertula. rioides, que son las más frecuentes, junto con las asociaciones de Wrangelia angus, Feldmannia irregularis y Bachelotia antillarum, que viven como epifitas de Padina spp., Dictyora y Coipomenia, además de encontrarse epífitas de otras algas en el borde del piso litoral.

EI epifitismo es alto y este proceso se observa en todas las áreas de estudio, cerca de un $40 \%$ de las especies encontradas correspondem a este tipo de relación biológica, algunas especies como: Achrochaetium dufourii, Erythorotrichia carnea, Gonio trichum alsidii. Sohacelaria furcigera. Giffordia duchassaigniana y Rhizoclonium spp., son epífitas estrictas pero nu manifiestan preferencia por algun biota en especial; Spermothamnion spelucarum solo se desarrolla en una especie en particular, constituyendo la relación Spermothamnion spelucarum-Bryothamnion seaforthï y otros más tienen un epifítismo facultativo entre los cuales se pueden citar a: Hypnea musciformis, Colpomenia sinuosa, Ectocarpus breviarticulatus, Sphacelaria tribuloides, Dictyota spp., Enteromorpha spp., Ulva lactucca y Cladophora fascicularis. Hay especies que soportan un grado alto de epífitas que en ocasiones enmascaran la presencia del epifitoda por ejemplo Digenia simplex, a la que se le aislaron más de 10 especies como epífitos; la sigue Sargassum vulgare que cuando habita el piso infralitoral en su parte más alta, modo expuesto sufre un alto epifitismo.

En general las bajas y altas que presentan ras poblaciones en las áreas rocosas de Veracruz no están bien marcadas a lo largo del año, el comportamiento de algunas especies comunes a las zonas tanto del eje como de la Sierra de los Tuxtlas, aparentemente anuales en Monte Pío, se manifiestan como'perennes, en el eje Neovolcánico tal es el caso de Cymopolia barbata y Laurencia obtusa. Las poblaciones de Ulva lactuca practicamente desaparecen durante los nortes o ciclones, pero la velocidad de recuperación es variable aún dentro de dos localidades cercanas como Villa Rica y Playa Paraíso, en la primera localidad tardan más en recuperarse, por su parte Ulva fasciata presente en Monte Pío desaparece durante el verano sin que este comportamiento haya estado relacionado con un temporal.

Por último se puede decir que las asociaciones comunes dominantes corresponden a: Sargassum vulgare, Sargassum vulgare-Corallina subulata, Ectocarpus brevearticulatus, Chnoospora minima y $E$. breviartuculatus-Ch. minima

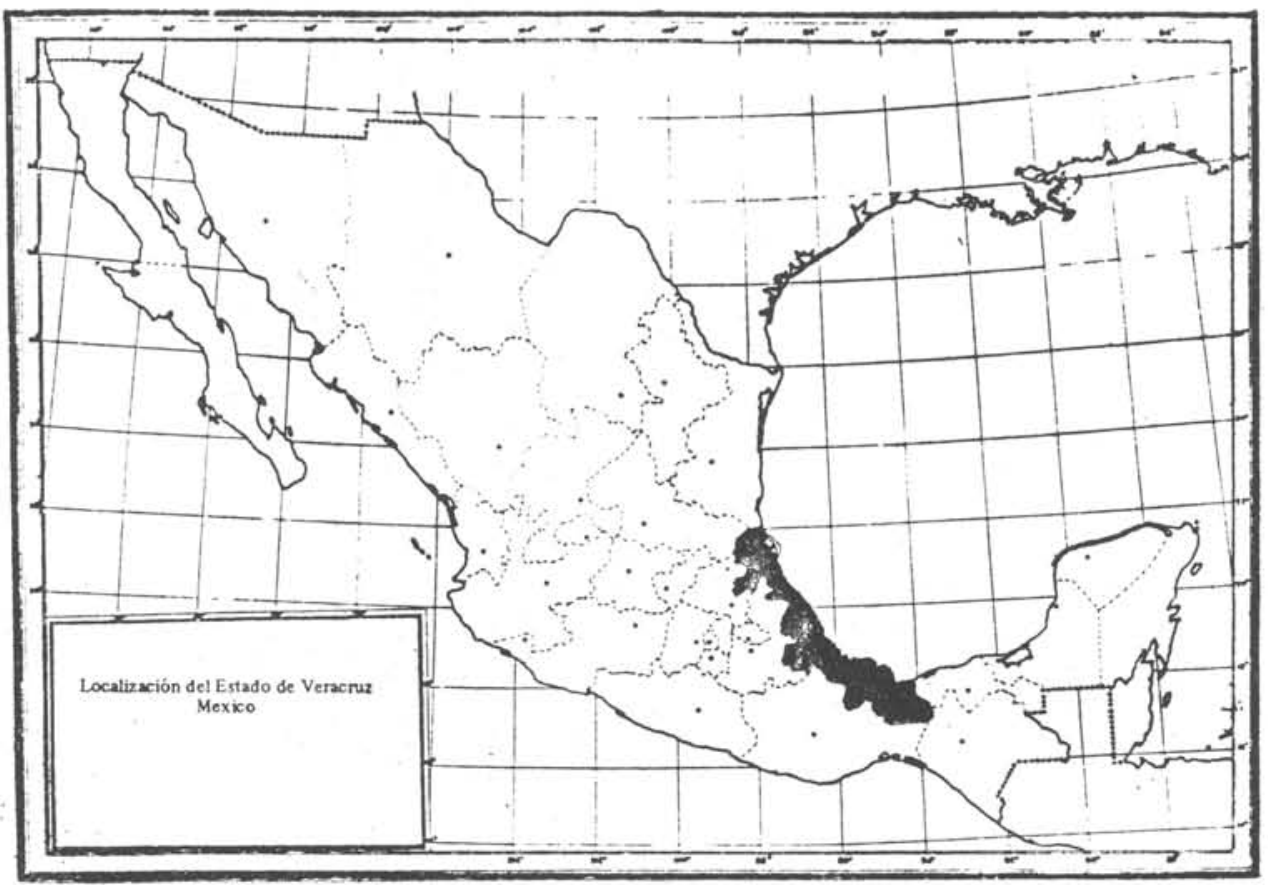


CUADRO

Lista de las especies encontradas en las localidades estudiadas

LOCALIDADES

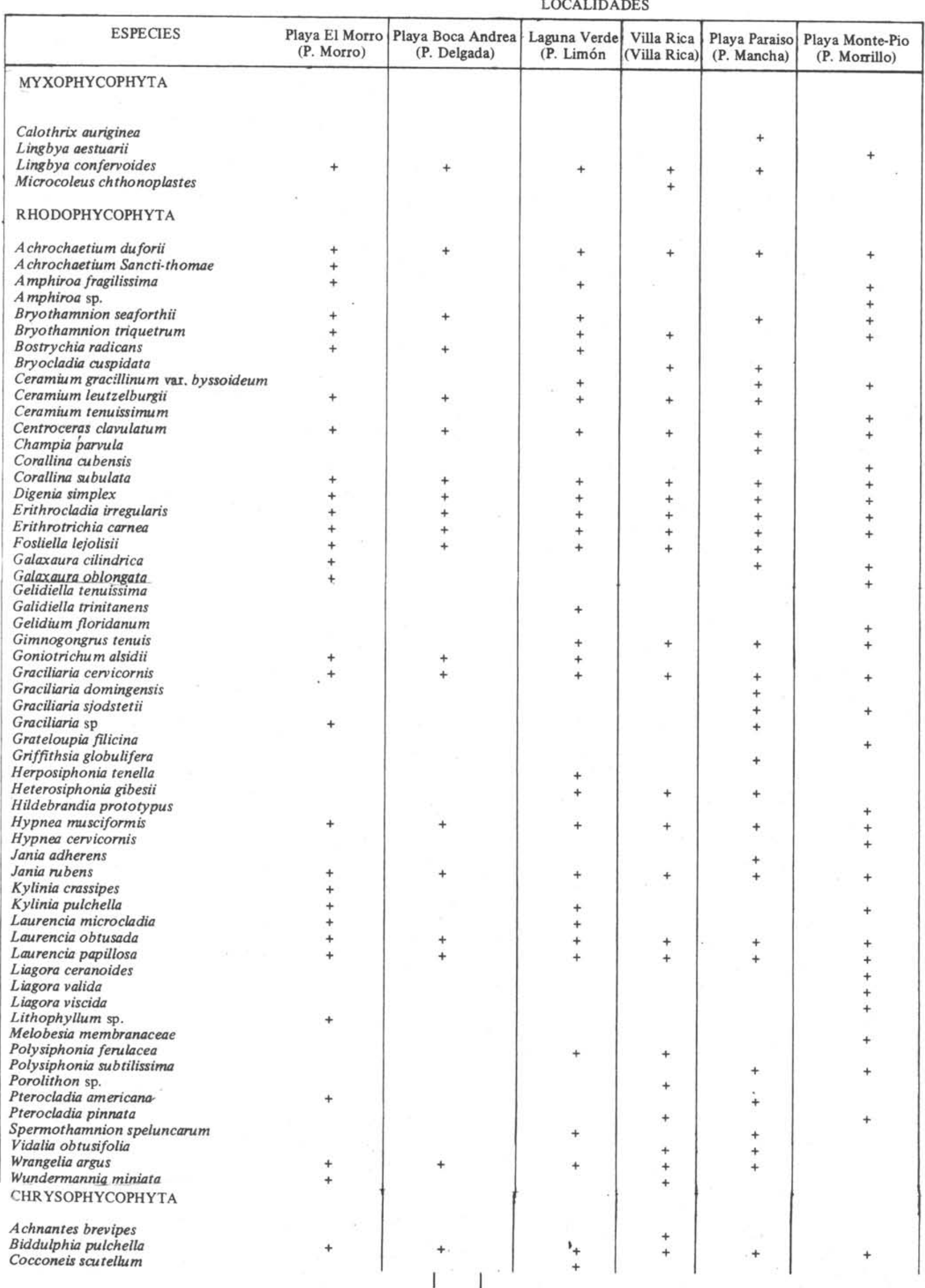


Gramathophora angulosa

Gramathophora marina

Melosira jurgensii

Melosira roeseana

Mavicula ramossisima

Nitzchia sp

\section{PHAEOPHYCOPHYTA}

A cinetospora crinito

Bachelotia antillarum

Chnoospora minima

Colpomenia sinuosa

Dictyopteris delicatula

Dictyota bartayresii

Dictyota cervicornis

Dictyota ciliolata

Dictyota dichotoma

Dictyota linearis

Dilophus Guineensis

Ectocarpus breviarticulatus

Ectocarpus conferuoides

Ectocarpus elachistaiform is

Ectocarpus variabilis

Feldmannia irregularis

Feldmannia padinae

Giffordia duchassaigniane

Giffordia mitchellae

Giffordia rallsiae

Padina gymnospora

Padina pavonica

Padina santae-crucis

Padina vickersiae

Ralfsia expansa

Rosenvingia intricata

Sargassum filipendule

Sargassum vulgare

Spatoglossum schoederii

Sphacelaria furcigera

Sphacelaria novae-hollandiae

Sphacelaria tribulcides

Stypopodium zonale

\section{CHLOROPHYCOPHYTA}

Anadyoneme stellata

Bryopsis pennata

Caulerpa racemosa var. laetevirens

Caulerpa racemosa var. macrophysia

Caulerpa racemosa var. uvifera

Caulerpa sertularioides

Caulerpa vickersia var. laetevirens

Chaetomorpha antenina

Chaetomorpha media

Cladophora fascicularis

Cladophora prolifera

Cladophoropsis membranacea

Codium taylori

Cymopolia barbata

Enteromorpha flexuosa

Enteromorpha lingulata

Enteromorpha salina

Halimeda opuntia

Halimeda tuna

$R$ hipocephalus phoenix

Rhizoclonium riparium

Rhizoclonium tortuosum

Ulva fasciata

Ulva lactuca

Ulvella lens

Valonia

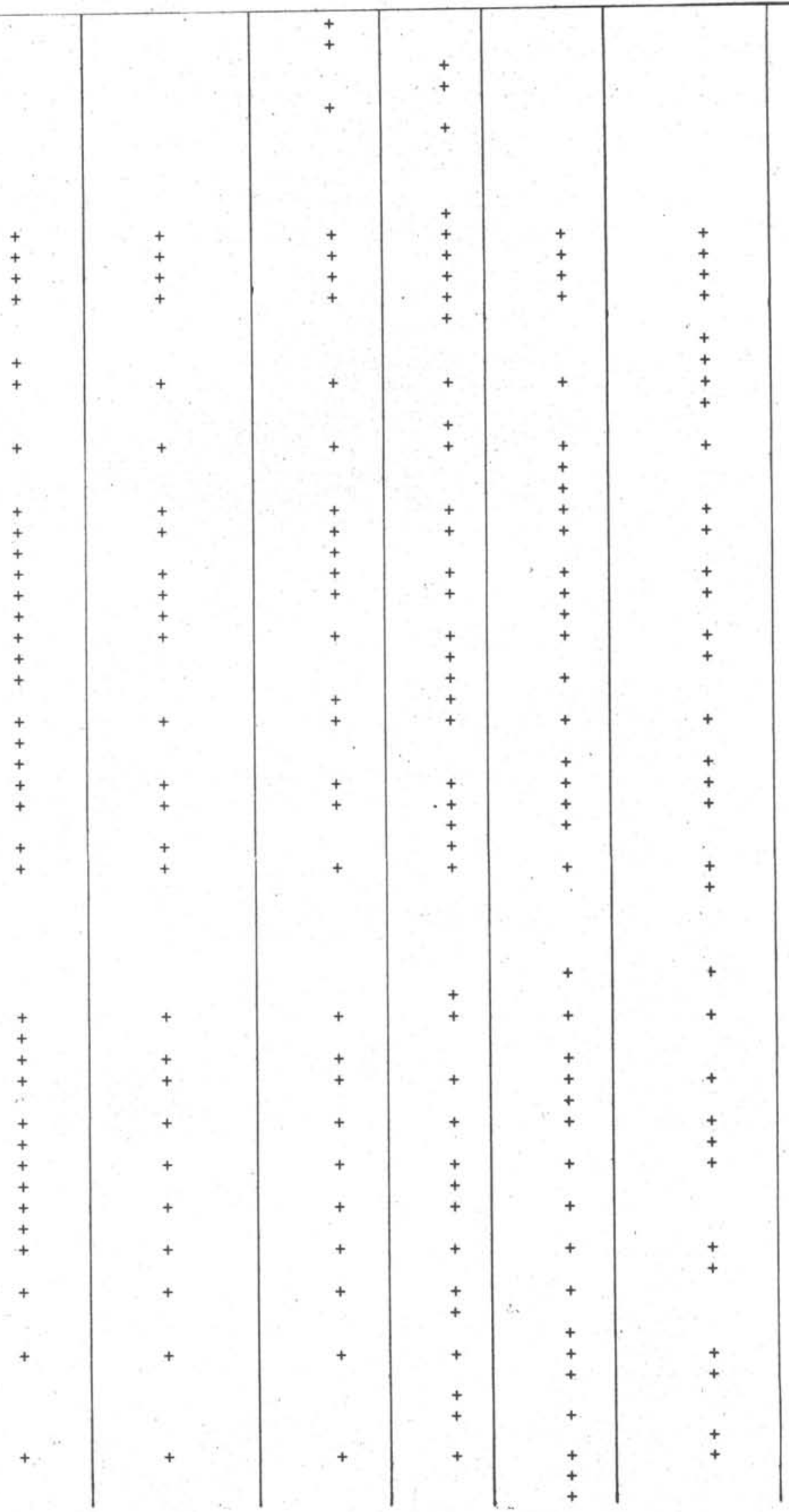

CUADRO II

\begin{tabular}{|c|c|c|c|c|c|c|c|c|c|}
\hline * & $\begin{array}{l}\text { TOTAL DE } \\
\text { ESPECIES }\end{array}$ & $\begin{array}{r}\text { ESPEC } \\
\text { TODAS LAS } \\
\text { LOCALIDADES }\end{array}$ & $\begin{array}{l}\text { ES COMUNES A: } \\
\text { LOCALIDADES } \\
\text { DEL EJE NEO- } \\
\text { VOLCANICO }\end{array}$ & $\begin{array}{c}\text { EL } \\
\text { MORRO }\end{array}$ & $\begin{array}{l}\text { BOCA } \\
\text { ANDREA }\end{array}$ & $\begin{array}{l}\text { ESPECIE } \\
\text { LAGUNA } \\
\text { VERDE }\end{array}$ & $\begin{array}{l}\text { S EXCLL } \\
\text { VILLA } \\
\text { RICA }\end{array}$ & $\begin{array}{c}\text { USIVAS DE: } \\
\text { PLAYA } \\
\text { PARAISO }\end{array}$ & MONTEPIO \\
\hline $\begin{array}{l}\text { CIANOFITAS } \\
\text { RODOFITAS } \\
\text { CRISOFITAS } \\
\text { FEOFITAS } \\
\text { CIDROFITAS }\end{array}$ & $\begin{array}{c}4 \\
58 \\
9 \\
33 \\
26\end{array}$ & $\begin{array}{c}0 \\
12 \\
1 \\
15 \\
5\end{array}$ & $\begin{array}{l}1 \\
5 \\
0 \\
0 \\
8\end{array}$ & $\begin{array}{l}0 \\
4 \\
0 \\
1 \\
1\end{array}$ & $\begin{array}{l}0 \\
0 \\
0 \\
0 \\
0\end{array}$ & $\begin{array}{l}0 \\
3 \\
4 \\
0 \\
0\end{array}$ & $\begin{array}{l}1 \\
1 \\
3 \\
3 \\
2\end{array}$ & $\begin{array}{l}1 \\
4 \\
0 \\
1 \\
2\end{array}$ & $\begin{array}{l}1 \\
6 \\
0 \\
1 \\
2\end{array}$ \\
\hline
\end{tabular}

